\title{
Habitual Caffeine Consumption Does Not Affect the Ergogenicity of Coffee Ingestion During a 5 km Cycling Time Trial
}

\author{
Neil D. Clarke and Darren L. Richardson \\ Coventry University
}

\begin{abstract}
There is growing evidence that caffeine and coffee ingestion prior to exercise provide similar ergogenic benefits. However, there has been a long-standing paradigm that habitual caffeine intake may influence the ergogenicity of caffeine supplementation. The aim of the present study was to investigate the effect of habitual caffeine intake on 5-km cycling time-trial performance following the ingestion of caffeinated coffee. Following institutional ethical approval, in a double-blind, randomized, crossover, placebocontrolled design, 46 recreationally active participants ( 27 men and 19 women) completed a 5-km cycling time trial on a cycle ergometer $60 \mathrm{~m}$ in following the ingestion of $0.09 \mathrm{~g} / \mathrm{kg}$ coffee providing $3 \mathrm{mg} / \mathrm{kg}$ of caffeine, or a placebo. Habitual caffeine consumption was assessed using a caffeine consumption questionnaire with low habitual caffeine consumption defined as $<3$ and $\geq 6 \mathrm{mg} \cdot \mathrm{kg}^{-1} \cdot \mathrm{day}^{-1}$ defined as high. An analysis of covariance using habitual caffeine intake as a covariant was performed to establish if habitual caffeine consumption had an impact on the ergogenic effect of coffee ingestion. Sixteen participants were classified as high-caffeine users and 30 as low. Ingesting caffeinated coffee improved 5-km cycling time-trial performance by $8 \pm 12 \mathrm{~s} ; 95 \%$ confidence interval (CI) [5, 13]; $p<.001 ; d=0.30$, with low, $9 \pm 14 \mathrm{~s} ; 95 \%$ CI $[3,14] ; p=.002 ; d=0.18$, and high, $8 \pm 10 \mathrm{~s} ; 95 \%$ CI $[-1,17] ; p=.008 ; d=0.06$, users improving by a similar magnitude, $95 \%$ CI $[-12,12] ; p=.946 ; d=0.08$. In conclusion, habitual caffeine consumption did not affect the ergogenicity of coffee ingestion prior to a 5-km cycling time trial.
\end{abstract}

Keywords: exercise performance, ergogenic, individual responses, supplements

Caffeine (1,3,7-trimethylxanthine) is consumed daily by approximately $80 \%$ of the world's population (Ogawa \& Ueki, 2007). Furthermore, due to the ergogenic potential, urine caffeine data suggests that caffeine is widely ingested prior to competition (Aguilar-Navarro et al., 2019), and athletes regularly consume caffeine in the form of coffee (Desbrow \& Leveritt, 2006). However, the majority of the research to date typically focuses on the ingestion of caffeine, rather than coffee (Higgins, Straight, \& Lewis, 2016). Finally, the responses to caffeine are often variable (Spriet, 2014) and possibly affected by a number of factors such as training status, genotype, and habitual caffeine use (Pickering \& Grgic, 2019a).

Caffeine's primary ergogenic mechanisms are considered to arise from the antagonism of adenosine receptors leading to an increase in neurotransmitter release, motor unit firing rates, pain suppression, reduced fatigue, and improved neuromuscular performance (Davis \& Green, 2009; Graham, 2001). However, there has been a long-standing paradigm that habitual caffeine intake may influence the ergogenicity of caffeine supplementation (Sökmen et al., 2008), and thus coffee ingestion. Habitual caffeine ingestion is associated with an upregulation in the number of adenosine receptors (Fredholm, 1982), potentially reducing the efficacy of the blocking action of caffeine. Furthermore, Svenningsson et al. (1999) reported caffeine-tolerant rats had downregulated levels of adenosine $\mathrm{A}_{2 \mathrm{~A}}$ receptors and the corresponding mRNA in rostral parts of striatum, potentially affecting caffeine metabolism, and thus altering the ergogenic effect of coffee. Therefore, caffeine

The authors are with the Faculty of Health and Life Sciences, School of Life Sciences, Coventry University, Coventry, United Kingdom. Clarke (neil.clarke@ coventry.ac.uk) is corresponding author. habituation is commonly identified as a factor influencing the ergogenic effect of caffeine (Sökmen et al., 2008), although research provides conflicting findings (Pickering \& Kiely, 2019), and with few studies reporting blood or salivary caffeine concentrations, or assessing men and women.

To date, few studies report no effect of habitual caffeine intake on exercise performance following caffeine ingestion $(\leq 25$ vs. $>300 \mathrm{mg} /$ day [Dodd, Brooks, Powers, \& Tulley, 1991]; 58$351 \mathrm{mg} /$ day [Gonçalves et al., 2017]; $27 \pm 36$ vs. $358 \pm 210 \mathrm{mg} /$ day [Sabol, Grgic, \& Mikulic, 2019]; $14 \pm 17$ vs. $777 \pm 295 \mathrm{mg} /$ day [Tarnopolsky \& Cupido, 2000]). Similarly, Irwin et al. (2011) concluded that acute caffeine supplementation provides an ergogenic benefit in regular caffeine users regardless of any withdrawal period. In contrast, Bell and McLellan (2002) reported that nonusers $(<50 \mathrm{mg} /$ day $)$ exhibited a greater, and longer lasting, ergogenic effect following the ingestion of $5 \mathrm{mg} / \mathrm{kg}$ of caffeine than regular caffeine users $(\geq 300 \mathrm{mg} /$ day $)$. Furthermore, Lara et al. (2019) reported that the ergogenic effects of caffeine $\left(3 \mathrm{mg} \cdot \mathrm{kg}^{-1} \cdot \mathrm{day}^{-1}\right)$ after 20 days of consecutive ingestion to be lower than the first day of ingestion. In addition, Beaumont et al. (2017) demonstrated that the ergogenic effect of caffeine to be reduced after 28 days of caffeine ingestion (Days 1-7: $1.5 \mathrm{mg} / \mathrm{kg}$; Days 8-28: $3 \mathrm{mg} / \mathrm{kg}$ ), suggesting the development of caffeine tolerance. These findings provide equivocal evidence for the existence of a reduction in caffeine's ergogenicity with continuous ingestion. However, the variation in these findings could be due to different thresholds to categorize individuals and the different performance protocols. Consequently, the relationship between habitual caffeine use and performance is poorly understood (Filip, Wilk, Krzysztofik, \& Del Cosco, 2020), and no previous study has investigated the effect of caffeine habituation on the ergogenic effect of coffee ingestion. Therefore, the aim of the present study was to investigate the effect of habitual caffeine 
intake on 5-km cycling time-trial performance following the ingestion of caffeinated coffee.

\section{Methods}

In a double-blind, Latin-square randomized, crossover, placebocontrolled design, in which participants and treatments were the blocking factors, 46 recreationally active participants $(27$ men: [mean $\pm \mathrm{SD}$ ] age $=29 \pm 6$ years, height $=180 \pm 6 \mathrm{~cm}$, body mass $=78 \pm 12 \mathrm{~kg} ; \dot{\mathrm{VO}}_{2} \max =55 \pm 11 \mathrm{ml} \cdot \mathrm{kg}^{-1} \cdot \min ^{-1}$ and 19 women: age $=28 \pm 6$ years, height $=166 \pm 7 \mathrm{~cm}$, body mass $=$ $72 \pm 11 \mathrm{~kg} ; \dot{\mathrm{VO}}_{2} \max =41 \pm 9 \mathrm{ml} \cdot \mathrm{kg}^{-1} \cdot \mathrm{min}^{-1}$ ) completed a $5-\mathrm{km}$ cycling time trial on a cycle ergometer $60 \mathrm{~min}$ following the ingestion of $0.09 \mathrm{~g} / \mathrm{kg}$ coffee providing $3 \mathrm{mg} / \mathrm{kg}$ of caffeine, or a placebo (PLA). A caffeine dose of $3 \mathrm{mg} / \mathrm{kg}$ was ingested as this dose has been demonstrated to offer ergogenic effects (Pickering \& Grgic, 2019b). Participants were recreational cyclists and had consistently trained on average $2 \pm 1$ times per week for at least 1 year. Originally, 54 participants were recruited, but seven were excluded due to not fulfilling the caffeine consumption inclusion criteria. All trials were conducted at the same time of day (09:00-12:00) and were consistent for each participant in order to minimize circadian variation (Drust, Waterhouse, Atkinson, Edwards, \& Reilly, 2005). Women were regularly taking a monophasic oral contraceptive pill for at least 3 months prior to the first trial. To control for possible differences during the oral contraceptive cycle, testing was performed during Days 5-8 and 19-22 of the cycle, as no changes in energy metabolism have been reported between these time points (Lynch, De Vito, \& Nimmo, 2001). All procedures were undertaken in accordance with the Declaration of Helsinki and approved by Coventry University institutional ethics committee. Participants were made fully aware of the exact procedures, including any risks and benefits of participation in the study before providing written informed consent.

Habitual caffeine consumption was assessed using a caffeine consumption questionnaire and assessed caffeine content (Bühler et al., 2014), and based on the proposals by Filip et al. (2020), low users included naïve to mild habitual caffeine consumption defined as $<3 \mathrm{mg} \cdot \mathrm{kg}^{-1} \cdot$ day $^{-1}$ and $\geq 6 \mathrm{mg} \cdot \mathrm{kg}^{-1} \cdot$ day $^{-1}$ defined as high users. The doses were chosen in order to make sure that there was a clear distension between the two groups. In addition, each participant completed a 24-hr dietary record prior to the first trial; this was then photocopied and handed back to the participants so that the same diet could be repeated for subsequent trials. Participants were also instructed to abstain from caffeine, alcohol, and strenuous activity for at least $12 \mathrm{hr}$, which along with diet, was confirmed verbally prior to each trial. Nescafe original coffee (from the same batch; Nescafé Original, Nestlé, United Kingdom) was used and dissolved in $300 \mathrm{ml}$ of water $\left(58 \pm 3^{\circ} \mathrm{C}\right)$ and served in lidded cups. Participants were given a maximum of 10 min to consume the beverage, and the 60 -min rest period started once the cup was emptied (Figure 1). The PLA trial was water of the same volume and temperature with coffee flavor (Espresso Coffee Flavoring Compound; MSK Ingredients, Chesterfield, United Kingdom) and color (Brown Food Coloring, Lakeland, United Kingdom). The coffee and PLA samples were analyzed externally for their caffeine content (Laserchrom HPLC Laboratories Ltd, Rochester, United Kingdom) using a high-performance liquid chromatography method. The coffee sample provided $35.1 \mathrm{mg}$ of caffeine per $1 \mathrm{~g}$ of coffee, and the PLA contained no traces of caffeine. Based on this analysis, it was calculated that each participant consumed $0.09 \mathrm{~g} / \mathrm{kg}$ of coffee to achieve the $3 \mathrm{mg} / \mathrm{kg}$ of caffeine required.

Before the experimental trials, participants completed a graded exercise test to exhaustion on a cycle ergometer (Wattbike Pro; Wattbike Ltd, Nottingham, United Kingdom) and a familiarization of the 5-km time trial, performed on the same day. Prior to all trials, participants performed a 2-min pretest warm-up. The protocol consisted of $2 \mathrm{~min}$ of cycling at a self-selected power output, 2 min of cycling including three 6-s maximal sprints, and finished with 3 min of cycling at a self-selected power output (Bellinger \& Minahan, 2014). Following completion of the warm-up, there was a 60-s period where the participants sat passively before a standardized countdown to initiate the time trial. The participants then performed a $5-\mathrm{km}$ cycling time trial where they were instructed to complete the distance as fast as possible. During each time trial, participants had access to the distance remaining, and with the exception of verbal encouragement, no other information was provided. The gearing was self-selected by the participants on the Wattbike during the familiarization trial and then replicated during each time trial. Trials were separated by a minimum period of $48 \mathrm{hr}$ to ensure recovery and caffeine washout. In order to establish any learning effect, following completion of the experimental trials, $5-\mathrm{km}$ time to completion was compared between the familiarization trial and PLA trial of the main experimental using a paired samples $t$ test. No significant difference between

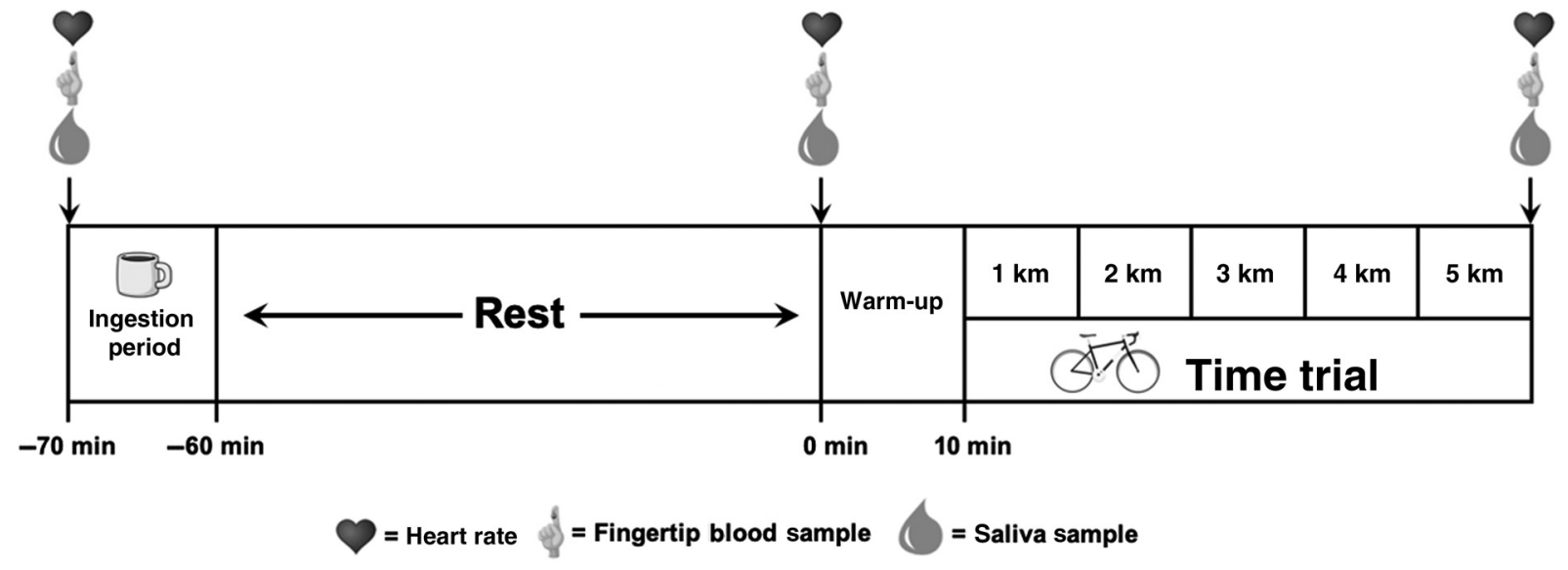

Figure 1 - Schematic of the experimental protocol. 
trials was observed, familiarization: $487 \pm 50 \mathrm{~s}$, PLA: $490 \pm 49 \mathrm{~s}$; $t(45)=0.265 ; p=.792 ; 95 \%$ confidence interval $(\mathrm{CI})[-19,25]$; Hedge's $g=-0.06,95 \%$ CI [ $-0.47,0.35]$.

Saliva samples (minimum $0.5 \mathrm{ml}$ by the passive drool technique) were obtained immediately before fluid ingestion, 1-hr postingestion prior to commencing the time trial, and $120 \mathrm{~s}$ following the time trial. Participants expectorated into a $20-\mathrm{ml}$ plastic cup, and the sample was then transferred to a capped glass vial that was immediately placed in $\mathrm{a}-80^{\circ} \mathrm{C}$ freezer for subsequent analysis of caffeine concentration using a standard enzyme-linked immunosorbent assay kit (Caffeine ELISA Kit; Creative Diagnostics, Shirley, NY). Saliva samples have been shown to be valid surrogates of plasma measures of caffeine in multiple studies (Scott, Chakraborty, \& Marks, 1984; Suzuki, Uematsu, Mizuno, Fujii, \& Nakashima, 1989). For example, data from Scott et al. (1984) revealed a strong correlation $(r=.94)$ between determinations of serum and saliva caffeine concentrations. Heart rate was measured throughout each time trial (Polar RS400; Polar Electro Oy, Kempele, Finland), and at the same time points, a capillary blood sample was drawn from the index finger for determination of blood lactate concentration (Biosen C-line; EKF-diagnostic $\mathrm{GmbH}$, Barleben, Germany).

A statistical power analysis was performed for sample size estimation based on the race time from a previous coffee-ingestion study (Clarke, Richardson, Thie, \& Taylor, 2018). As this study did not present correlation values between conditions, a conservative effect size value of 0.5 was used for the calculation. Consequently, an a priori power calculation suggested a sample size of 11 participants was deemed necessary to detect a difference between conditions given an estimated effect size of 0.44 , a $1-\beta$ error probability of .95 and an alpha value of $<.05$. A mixed-design 2 (between-subject factor; high-/low-caffeine users) $\times 2$ (withinsubject factor; condition: caffeinated coffee, PLA) an analysis of variance was applied. Where any differences were identified, post hoc pairwise comparisons with Bonferroni correction were conducted. In addition, an analysis of covariance was performed using habitual caffeine intake and baseline performance as covariates in order to further establish the ergogenic effect of coffee ingestion and the effect of habitual caffeine intake and initial performance. Linear regressions were performed between habitual caffeine

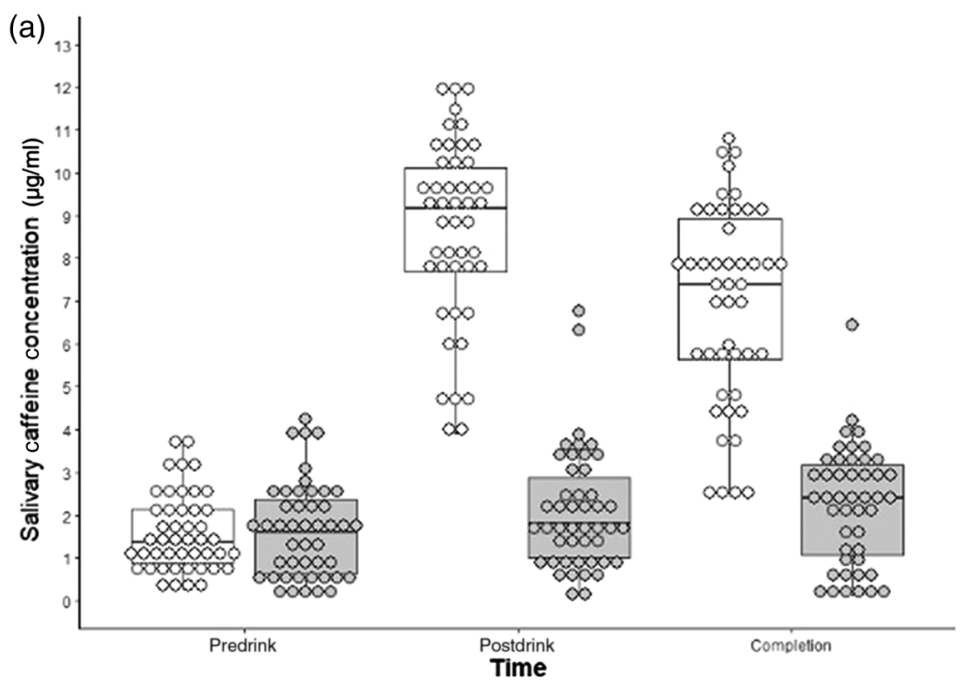

intake, postdrink salivary caffeine concentration, and the absolute change in performance. All data were analyzed using IBM SPSS Statistics for Windows (version 25.0; IBM Corp., Armonk, NY). Finally, 95\% CIs and effect sizes, (partial eta squared $\left[\eta_{\mathrm{p}}^{2}\right]$ ) defined as trivial $(<.10)$, small $(.10-.24)$, moderate $(.25-.39)$, or large ( $\geq .40)$, and Hedge's $g$ defined as trivial $(\leq 0.19)$, small (0.20-0.49), moderate $(0.50-0.79)$, and large $(\geq 0.80)$ according to the cutoffs suggested by Cohen (1992), were also calculated.

\section{Results}

A total of 16 participants (11 men and five women) were classified as high-caffeine users, $6 \pm 2 \mathrm{mg} \cdot \mathrm{kg}^{-1} \cdot$ day $^{-1}$; 95\% CI [5, 7]; $415 \pm 133 \mathrm{mg} / \mathrm{day} ; 95 \%$ CI [344, 486], and 30 as low, 16 men and 14 women; $2 \pm 1 \mathrm{mg} \cdot \mathrm{kg}^{-1} \cdot \mathrm{day}^{-1} ; 95 \% \mathrm{CI}[1,2] ; 153 \pm 99 \mathrm{mg} /$ day; $95 \%$ CI [116, 190]. Coffee ingestion increased salivary caffeine levels, $F(3,132)=278.553 ; p<.001 ; 95 \%$ CI [6.1, 7.9]; $\eta_{\mathrm{p}}^{2}=.86$ (Figure 2a), but with no difference between low, $7.3 \pm 2.3 \mu \mathrm{g} / \mathrm{ml} ; 95 \%$ CI $[6.5,8.0]$; Hedge's $g=4.13$, 95\% CI [3.25, 5.01], and high, $6.7 \pm 1.5 \mu \mathrm{g} / \mathrm{ml}$; 95\% CI [5.7, 7.8]; Hedge's $g=4.88,95 \%$ CI $[3.35,6.14]$, users, $F(1,44)=1.017 ; p=.319$; $95 \%$ CI $[-0.4,1.2] ; \eta_{\mathrm{p}}^{2}=.02$ (Figure $2 \mathrm{~b}$ ).

Overall, performance in the coffee trial $(482 \pm 46 \mathrm{~s})$ was faster than the PLA, $490 \pm 49 \mathrm{~s} ; t(45)=-4.523 ; p<.001 ; 95 \%$ CI $[-12$, -5]; Hedge's $g=0.17,95 \%$ CI $[-0.24,0.56]$, trial. Low-caffeine users (Coffee: $477 \pm 49$ s; PLA $486 \pm 52$ s) and high users performed similarly, Coffee: $490 \pm 40 \mathrm{~s} ;$ PLA $497 \pm 0$. s; $F(1$, $44)=0.679 ; p=.415 ; 95 \%$ CI $\left.[-41,-17] ; \eta_{p}^{2}=.02\right)$. Ingesting coffee improved $5-\mathrm{km}$ cycling time-trial performance by $8 \pm 12$ s, 95\% CI [5, 13]; $F(1,44)=18.032 ; p<.001 ; \eta_{\mathrm{p}}^{2}=.30$ (Figure 3a), with low, $9 \pm 14$ s; 95\% CI [3, 14]; $p=.002$; Hedge's $g=0.18,95 \%$ CI $[-0.33,0.68]$, and high users, $8 \pm 10 \mathrm{~s} ; 95 \%$ CI $[-1,17] ; p=.008 ;$ Hedge's $g=0.17,95 \%$ CI $[-0.53,0.86]$, improving by a similar magnitude, $95 \%$ CI $[-12,12] ; p=.946$; Hedge's $g=0.07,95 \%$ CI [-0.54, 0.68] (Figure 3b). Analysis of covariance revealed no influence of habitual caffeine intake on exercise performance, $F(1,43)=0.013 ; p=.910 ; \eta_{\mathrm{p}}^{2}=.00$. Men (COF: $451 \pm 31 \mathrm{~s}$; PLA $465 \pm 38 \mathrm{~s}$ ) completed the time trial moderately quicker than women, COF: $516 \pm 42$ s; PLA $526 \pm$ $40 \mathrm{~s} ; F(1,41)=0.984 ; p=.761 ; 95 \%$ CI $[-85,-38] ; \eta_{\mathrm{p}}^{2}=.40$.

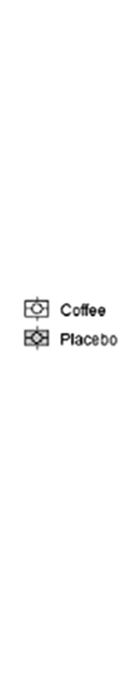

Figure 2 - (a) Distribution and individual salivary caffeine responses during the 5-km cycling time trial. (b) Individual changes in salivary caffeine concentration following coffee ingestion in habitually low- and high-caffeine users. 

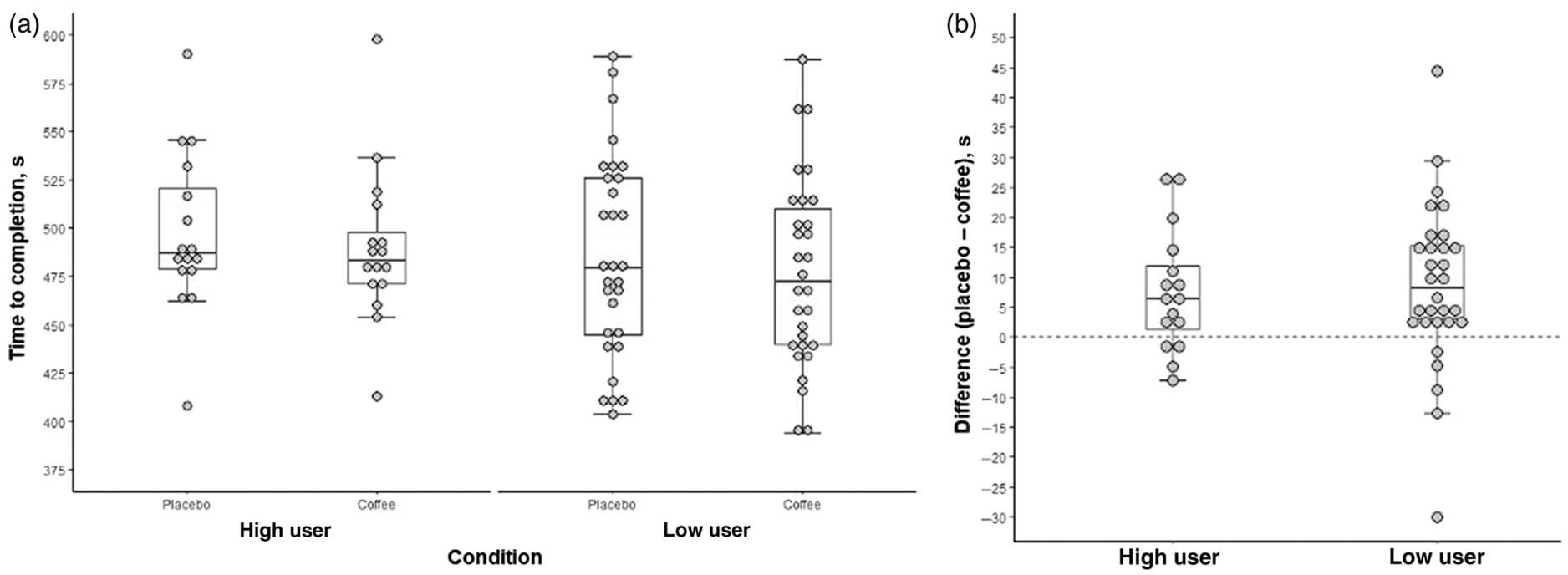

Figure 3 - (a) Distribution and individual 5-km cycling time-trial time for completion in habitually low- and high-caffeine users. (b) Individual changes in time-trial duration (placebo time minus coffee time, where positive values represent a faster time following the ingestion of coffee) in habitually low- and high-caffeine users.

However, no significant sex and trial interaction was observed, $F(1$, $41)=0.504 ; p=.482 ; \eta_{\mathrm{p}}^{2}=.01$, with men and women both improving by approximately 8 and $9 \mathrm{~s}$, respectively, following coffee ingestion, and with only a Trivial trial $\times$ Sex $\times$ Habitual caffeine intake interaction, $F(1,41)=0.984 ; p=.327 ; \eta_{\mathrm{p}}^{2}=.02$. Furthermore, in order to account for baseline performance (PLA) between low and high users, an analysis of covariance was performed using the PLA condition as a covariant, which demonstrated no significant difference in the performance following the ingestion of coffee, $F(1,43)=0.708 ; p=.405 ; \eta_{\mathrm{p}}^{2}=.02$, and as such, both groups improved by a similar magnitude (low users: $1.7 \pm 2.8 \%$; high users: $1.8 \pm 2.0 \%$ ). In addition, no order effect was observed, $t(45)=0.253 ; p=.802$; Hedge's $g=0.04,95 \%$ CI $[-0.37,0.44]$. Finally, no association was observed between habitual caffeine intake $\left(r^{2}=.002 ; p=.828\right.$; Figure $4 \mathrm{a})$ or postdrink salivary caffeine concentration $\left(r^{2}=.002\right.$; $p=.188$; Figure 4b) and time-trial performance following coffee ingestion.

A small condition and time interaction for blood lactate was observed, $F(2,88)=6.818 ; p=.002 ; \eta_{\mathrm{p}}^{2}=.13$ (Table 1$)$, with values higher at the completion of COF compared with PLA, $p=.001 ; 95 \%$ CI $[0.5,1.8]$; Hedge's $g=0.42$, 95\% CI [0.01, $0.84]$. However, there were no differences observed between low- and high-caffeine users, $F(1,44)=0.163 ; p=.688 ; \eta_{\mathrm{p}}^{2}=.00$. Similarly, a small increase heart rate was observed during $\mathrm{COF}$ compared with PLA, $F(1,41)=6.296 ; p=.016$; 95\% CI $[1,5]$; $\eta_{\mathrm{p}}^{2}=.13$ (Table 1 ), although only trivial differences, $F(1,41)=$ $0.829 ; p=.368 ; 95 \%$ CI $[-9,3] ; \eta_{\mathrm{p}}^{2}=.02$ (Table 1 ), in heart rate were observed between low- and high-caffeine users.

\section{Discussion}

The aim of the present study was to investigate the effect of habitual caffeine intake on 5-km cycling time-trial performance following the ingestion of coffee. Ingesting coffee, providing $3 \mathrm{mg} / \mathrm{kg}$ of caffeine improved 5-km cycling time-trial performance with similar performance observed between habitually low- and high-caffeine users. Consequently, habitual caffeine consumption did not affect the ergogenic effect of coffee ingestion prior to a $5-\mathrm{km}$ cycling time trial.

Ingesting coffee reduced $5-\mathrm{km}$ cycling time-trial completion time by $1.7 \pm 2.6 \%(8 \pm 12 \mathrm{~s})$, with low users improving by $1.7 \pm 2.8 \%(9 \pm 14 \mathrm{~s})$ and high users by $1.8 \pm 2.0 \%(8 \pm 10 \mathrm{~s})$. These findings support Clarke et al. (2019), who observed that coffee providing $3 \mathrm{mg} / \mathrm{kg}$ of caffeine improved $5-\mathrm{km}$ cycling timetrial performance by $1.9 \%$ (men: $2.1 \%$ and women: $1.8 \%$ ) compared with a PLA and Clarke et al. (2018) where the ingestion of caffeinated coffee providing $3 \mathrm{mg} / \mathrm{kg}$ of caffeine improved 1-mile race time by $1.9 \%$ compared with a PLA. In addition, Wiles et al. (1992) reported that the ingestion of $3 \mathrm{~g}$ of caffeinated coffee, containing approximately 150-200 $\mathrm{mg}$ of caffeine, improved $1500-\mathrm{m}$ treadmill running performance by $4.2 \mathrm{~s}(1.4 \%)$ when compared with decaffeinated coffee. Similarly, Hodgson et al. (2013) demonstrated that coffee and caffeine $(5 \mathrm{mg} / \mathrm{kg})$ ingestion improved time-trial performance by approximately 5\%. Despite these overall observations, individual performance responses to coffee ingestion do exist as highlighted in Figure 3b; that is, the magnitude of performance change varies between individuals $(-7.3 \%$ to $8.1 \%)$. Consequently, habitual caffeine intake is a potential factor for the underlying heterogeneous responses (Bell \& McLellan, 2002). However, empirically this suggestion is not conclusive; three studies have reported a blunting of caffeine's acute ergogenic effects with habitual use (Beaumont et al., 2017; Bell \& McLellan, 2002; Lara et al., 2019), and two reported no differences in response to acute caffeine ingestion between individuals with different habitual caffeine intakes (Dodd et al., 1991; Gonçalves et al., 2017). The present study suggests there is no association between habitual caffeine intake and the absolute change in the 5-km time-trial performance (Figure 4a), refuting the suggestion that habitual caffeine intake might reduce the ergogenic effect of coffee ingestion. One possible explanation for this occurrence is the limited association between preexercise salivary caffeine concentration and exercise performance (Figure 4b). Therefore, the findings of the present study add to the body of evidence suggesting that habitual caffeine consumption does not negatively affect the ergogenic effect of caffeine or coffee. 

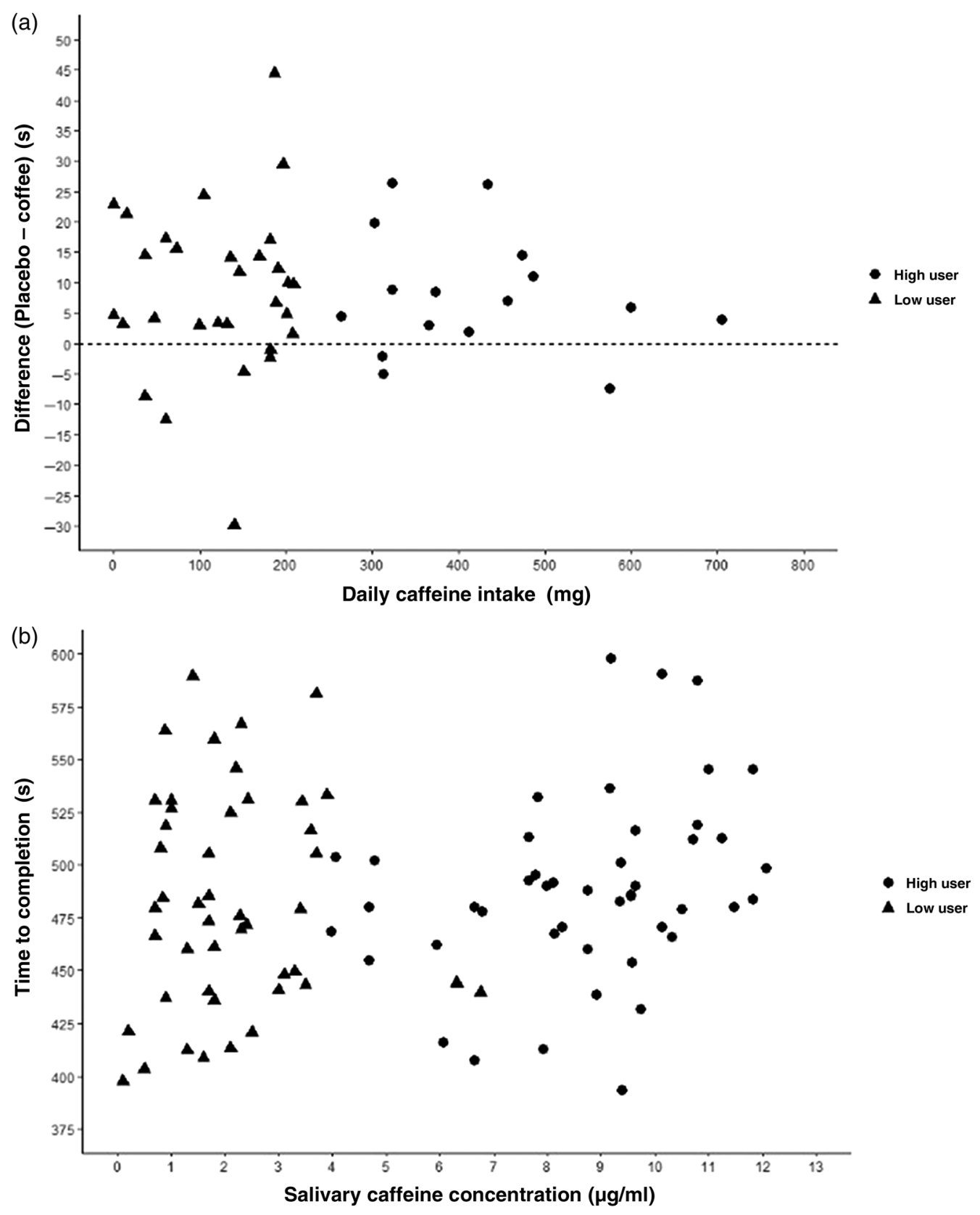

Figure 4 - (a) Relationship between individual changes in time-trial duration (placebo - coffee, where positive values represents a faster time following the ingestion of coffee) and habitual daily caffeine intake. (b) Relationship between postdrink salivary caffeine concentration and time-trial duration.

However, it should be noted that the concept that habitual caffeine intake moderates the acute ergogenic effects of caffeine supplementation was derived from animal studies with very high doses (i.e., $20 \mathrm{mg} / \mathrm{kg}$ ) (Fredholm, 1982), and as such may not be relevant to humans.

The metabolism of caffeine in the liver is largely dependent on the CYP1A2 isoform of cytochrome 450 and Djordjevic et al. (2010) reported that the daily consumption of at least 3 cups of coffee, defined as "heavy drinkers," increased CYP1A2 activity potentially increasing caffeine metabolism. Furthermore, Svenningsson et al. (1999) reported alterations in gene expression within the striatum albeit in rats, which may be crucial in the development of tolerance to caffeine, potentially affecting caffeine metabolism, and thus altering the ergogenic effect of caffeine and coffee. When specifically examining exercise of the nature in the present study, the primary mechanisms by which caffeine exerts its ergogenic effects are considered to arise from the antagonism of adenosine receptors leading to an increase in neurotransmitter release and motor unit firing rates, pain suppression, reduced fatigue, and improved neuromuscular performance (Graham, 2001). Furthermore, Meeusen et al. (2013) suggested that both motor effects and motivational aspects increase when caffeine blocks adenosine receptors, creating a greater dopaminergic drive, and thus enhancing 5-km performance. However, data suggest that habitual intake of caffeine is associated with an increased number of adenosine receptors (Fredholm, 1982) attenuating caffeine's 
Table 1 Mean \pm SD Heart Rate and Blood Lactate Concentrations During the 5-Km Cycling Time Trial in Habitually Low- and High-Caffeine Users

\begin{tabular}{|c|c|c|c|c|c|c|}
\hline \multirow[b]{2}{*}{ Variable } & \multicolumn{3}{|c|}{ Placebo } & \multicolumn{3}{|c|}{ Coffee } \\
\hline & Predrink & Postdrink & Completion & Predrink & Postdrink & Completion \\
\hline \multicolumn{7}{|c|}{ Heart rate (beats per minute) } \\
\hline Overall & $69 \pm 12$ & $68 \pm 13$ & $171 \pm 12$ & $71 \pm 14$ & $70 \pm 13$ & $180 \pm 11$ \\
\hline Low user & $71 \pm 14$ & $68 \pm 14$ & $173 \pm 12$ & $71 \pm 15$ & $71 \pm 15$ & $179 \pm 11$ \\
\hline High user & $71 \pm 10$ & $73 \pm 10$ & $178 \pm 12$ & $74 \pm 10$ & $74 \pm 10$ & $182 \pm 11$ \\
\hline \multicolumn{7}{|c|}{ Blood lactate (mmol/L) } \\
\hline Overall & $1.9 \pm 0.8$ & $2.3 \pm 1$ & $12.0 \pm 2.8$ & $2.1 \pm 0.9$ & $2.6 \pm 1.2$ & $13.2 \pm 2.8$ \\
\hline Low user & $2.0 \pm 0.9$ & $2.3 \pm 1.1$ & $12.3 \pm 2.8$ & $2.2 \pm 1.0$ & $2.5 \pm 1.1$ & $13.1 \pm 2.6$ \\
\hline High user & $1.7 \pm 0.4$ & $2.3 \pm 0.8$ & $11.4 \pm 3.0$ & $2.0 \pm 0.9$ & $2.9 \pm 1.4$ & $13.4 \pm 3.3$ \\
\hline
\end{tabular}

stimulatory effects (Svenningsson et al., 1999). In addition, a caffeine-induced increase in the secretion of $\beta$-endorphins (Laurent et al., 2000) may be a potential mechanism via which, caffeine attenuates pain sensation and rating of perceived exertion during exercise, thereby decreasing perceptions of effort and/or improving performance, as observed in the present study. However, Haskell et al. (2005) reported that the ingestion of caffeine tended to improve mood and cognitive performance more in lowcaffeine users compared high. Consequently, while habitual caffeine consumption may cause alterations in caffeine metabolism, the efficacy of adenosine antagonism and mood, the evidence from the present study suggests that they do not translate into impaired exercise performance in high-caffeine users.

On a practical level, Lara et al. (2019) reported that there is a progressive tolerance to caffeine ingestion, and there are suggestions that the preexercise caffeine dose needs to exceed the level of habitual intake (Pickering \& Kiely, 2018). However, the present study showed that the level of habitual caffeine ingestion was not associated with the magnitude of improvement in $5-\mathrm{km}$ performance following the ingestion of coffee providing $3 \mathrm{mg} / \mathrm{kg}$ of caffeine, a value below the habitual intake of the high users $\left(6 \pm 2 \mathrm{mg} \cdot \mathrm{kg}^{-1} \cdot \mathrm{day}^{-1}\right)$. Furthermore, Pickering and Kiely (2019) concluded that short-term precompetition caffeine withdrawal appears to offer little benefit, and given the potential negative side effects, withdrawal strategies are not recommended. In addition, Irwin et al. (2011) concluded that acute caffeine supplementation positively effects exercise performance and provides an ergogenic benefit in regular caffeine users regardless of any withdrawal period. Therefore, consuming coffee providing $3 \mathrm{mg} / \mathrm{kg} 60 \mathrm{~min}$ prior to exercise is a practical source of caffeine prior to exercise in habitual low- and high-caffeine users. However, given the individual response to caffeine, athletes should experiment with various doses and timing strategies when using caffeine to enhance performance (Pickering \& Kiely, 2019).

The present study is not without limitations. There are difficulties with relying on self-report caffeine consumption questionnaires in order to determine habitual use of caffeine as participants may not be fully aware of what products contain caffeine, and the caffeine content in drinks can vary between caffeine sources and time points (Desbrow et al., 2007). Furthermore, the use of a 24-hr dietary record to ensure pretrial standardization, while being an acceptable method, under reporting can occur (Poslusna, Ruprich, de Vries, Jakubikova, \& van't Veer, 2009). In addition, the baseline salivary caffeine concentrations suggest that possibly not all participants abstained from caffeine for $12 \mathrm{hr}$ prior to each trial.
However, both factors are likely to provide a more ecologically valid condition and represent typical precompetition preparation. Furthermore, there are also important strengths to this study, namely the caffeine content of the coffee and salivary caffeine concentration has been assessed.

\section{Conclusions}

Habitual caffeine consumption did not affect the ergogenic effect of coffee ingestion during $5-\mathrm{km}$ cycling time trial. Furthermore, ingesting coffee providing $3 \mathrm{mg} / \mathrm{kg}$ of caffeine increased salivary caffeine levels and improved 5-km cycling time-trial performance, and thus, coffee is a practical source of caffeine. However, the underlying mechanisms are not fully elucidated, and given the limitations listed, further investigations are required to understand the impact of habitual caffeine ingestion on the ergogenic effect of coffee and caffeine ingestion.

\section{Acknowledgments}

First, the authors would like to offer their thanks to all participants who completed the study. Neil D. Clarke contributed toward the original research idea and development of the experimental design. All authors contributed toward various aspects of data collection, sample collection, and analysis. All authors aided with the writing, reading, and approval of the final version of this original manuscript. Neil D. Clarke has previously received funding from the Institute for Scientific Information on Coffee (ISIC).

\section{References}

Aguilar-Navarro, M., Muñoz, G., Salinero, J.J., Muñoz-Guerra, J., Fernández-Álvarez, M., Plata, M.D.M., \& Del Coso, J. (2019). Urine caffeine concentration in doping control samples from 2004 to 2015. Nutrients, 11(2), 286. doi:10.3390/nu11020286

Beaumont, R., Cordery, P., Funnell, M., Mears, S., James, L., \& Watson, P. (2017). Chronic ingestion of a low dose of caffeine induces tolerance to the performance benefits of caffeine. Journal of Sports Sciences, 35(19), 1920-1927. PubMed ID: 27762662 doi:10.1080/ 02640414.2016 .1241421

Bell, D.G., \& McLellan, T.M. (2002). Exercise endurance 1, 3, and 6 h after caffeine ingestion in caffeine users and nonusers. Journal of Applied Physiology, 93(4), 1227-1234. doi:10.1152/japplphysiol. 00187.2002 
Bellinger, P.M., \& Minahan, C. (2014). Reproducibility of a laboratory based 1-km Wattbike cycling time trial in competitive cyclists. Journal of Science and Cycling, 3(3), 23-28.

Bühler, E., Lachenmeier, D., Schlegel, K., \& Winkler, G. (2014). Development of a tool to assess the caffeine intake among teenagers and young adults. Ernahrungs Umschau, 61(4), 58-63.

Clarke, N.D., Kirwan, N.A., \& Richardson, D.L. (2019). Coffee ingestion improves $5 \mathrm{~km}$ cycling performance in men and women by a similar magnitude. Nutrients, 11(11), 2575. doi:10.3390/nu11112575

Clarke, N.D., Richardson, D.L., Thie, J., \& Taylor, R. (2018). Coffee ingestion enhances 1-mile running race performance. International Journal of Sports Physiology and Performance, 13(6), 789-794. PubMed ID: 29140142 doi:10.1123/ijspp.2017-0456

Cohen, J. (1992). A power primer. Psychological Bulletin, 112(1), 155159. PubMed ID: 19565683 doi:10.1037/0033-2909.112.1.155

Davis, J.K., \& Green, J.M. (2009). Caffeine and anaerobic performance: Ergogenic value and mechanisms of action. Sports Medicine, 39(10), 813-832. PubMed ID: 19757860 doi:10.2165/11317770-00000000000000

Desbrow, B., Hughes, R., Leveritt, M., \& Scheelings, P. (2007). An examination of consumer exposure to caffeine from retail coffee outlets. Food and Chemical Toxicology, 45(9), 1588-1592. PubMed ID: 17412475 doi:10.1016/j.fct.2007.02.020

Desbrow, B., \& Leveritt, M. (2006). Awareness and use of caffeine by athletes competing at the 2005 Ironman Triathlon World Championships. International Journal of Sport Nutrition and Exercise Metabolism, 16(5), 545-558. PubMed ID: 17240785 doi:10.1123/ijsnem. 16.5 .545

Djordjevic, N., Ghotbi, R., Jankovic, S., \& Aklillu, E. (2010). Induction of CYP1A2 by heavy coffee consumption is associated with the CYP1A2 -163C>A polymorphism. European Journal of Clinical Pharmacology, 66(7), 697-703. PubMed ID: 20390257 doi:10.1007/ s00228-010-0823-4

Dodd, S.L., Brooks, E., Powers, S.K., \& Tulley, R. (1991). The effects of caffeine on graded exercise performance in caffeine naive versus habituated subjects. European Journal of Applied Physiology and Occupational Physiology, 62(6), 424-429. PubMed ID: 1893906 doi:10.1007/BF00626615

Drust, B., Waterhouse, J., Atkinson, G., Edwards, B., \& Reilly, T. (2005). Circadian rhythms in sports performance-An update. Chronobiology International, 22(1), 21-44. PubMed ID: 15865319 doi:10.1081/ CBI-200041039

Filip, A., Wilk, M., Krzysztofik, M., \& Del Coso, J. (2020). Inconsistency in the ergogenic effect of caffeine in athletes who regularly consume caffeine: Is it due to the disparity in the criteria that defines habitual caffeine intake? Nutrients, 12(4), 1087. doi:10.3390/nu12041087

Fredholm, B.B. (1982). Adenosine actions and adenosine receptors after 1 week treatment with caffeine. Acta Physiologica Scandinavica, 115(2), 283-286. PubMed ID: 6291335 doi:10.1111/j.1748-1716. 1982.tb07078.x

Gonçalves, L.S., Painelli, V.S., Yamaguchi, G., Oliveira, L.F., Saunders, B., da Silva, R.P., ... Gualano, B. (2017). Dispelling the myth that habitual caffeine consumption influences the performance response to acute caffeine supplementation. Journal of Applied Physiology, 123(1), 213-220. doi:10.1152/japplphysiol.00260.2017

Graham, T.E. (2001). Caffeine and exercise: Metabolism, endurance and performance. Sports Medicine, 31(11), 785-807. PubMed ID: 11583104 doi:10.2165/00007256-200131110-00002

Haskell, C.F., Kennedy, D.O., Wesnes, K.A., \& Scholey, A.B. (2005). Cognitive and mood improvements of caffeine in habitual consumers and habitual non-consumers of caffeine. Psychopharmacology, 179(4), 813-825. doi:10.1007/s00213-004-2104-3
Higgins, S., Straight, C.R., \& Lewis, R.D. (2016). The effects of preexercise caffeinated coffee ingestion on endurance performance: An evidence-based review. International Journal of Sport Nutrition and Exercise Metabolism, 26(3), 221-239. PubMed ID: 26568580 doi:10. 1123/ijsnem.2015-0147

Hodgson, A.B., Randell, R.K., \& Jeukendrup, A.E. (2013). The metabolic and performance effects of caffeine compared to coffee during endurance exercise. PLoS One, 8(4), e59561. PubMed ID: 23573201 doi:10.1371/journal.pone.0059561

Irwin, C., Desbrow, B., Ellis, A., O’Keeffe, B., Grant, G., \& Leveritt, M. (2011). Caffeine withdrawal and high-intensity endurance cycling performance. Journal of Sports Sciences, 29(5), 509-515. PubMed ID: 21279864 doi:10.1080/02640414.2010.541480

Lara, B., Ruiz-Moreno, C., Salinero, J.J., \& Del Coso, J. (2019). Time course of tolerance to the performance benefits of caffeine. PLoS One, 14(1), e210275. PubMed ID: 30673725 doi:10.1371/journal.pone. 0210275

Laurent, D., Schneider, K.E., Prusaczyk, W.K., Franklin, C., Vogel, S.M., Krssak, M., ... Shulman, G.I. (2000). Effects of caffeine on muscle glycogen utilization and the neuroendocrine axis during exercise. The Journal of Clinical Endocrinology and Metabolism, 85(6), 21702175. PubMed ID: 10852448 doi:10.1210/jcem.85.6.6655

Lynch, N.J., De Vito, G., \& Nimmo, M.A. (2001). Low dosage monophasic oral contraceptive use and intermittent exercise performance and metabolism in humans. European Journal of Applied Physiology, 84(4), 296-301. PubMed ID: 11374113 doi:10.1007/s004210000380

Meeusen, R., Roelands, B., \& Spriet, L.L. (2013). Caffeine, exercise and the brain. Nestle Nutrition Institute Workshop Series, 76, 1-12. PubMed ID: 23899750 doi:10.1159/000350223

Ogawa, N., \& Ueki, H. (2007). Clinical importance of caffeine dependence and abuse. Psychiatry and Clinical Neurosciences, 61(3), 263-268. PubMed ID: 17472594 doi:10.1111/j.1440-1819.2007.01652.x

Pickering, C., \& Grgic, J. (2019a). Caffeine and exercise: What next? Sports Medicine, 49(7), 1007-1030. doi:10.1007/s40279-019-01 101-0

Pickering, C., \& Grgic, J. (2019b). Is coffee a useful source of caffeine preexercise? International Journal of Sport Nutrition and Exercise Metabolism, 30(1), 69-82. doi:10.1123/ijsnem.2019-0092

Pickering, C., \& Kiely, J. (2018). Are the current guidelines on caffeine use in sport optimal for everyone? Inter-individual variation in caffeine ergogenicity, and a move towards personalised sports nutrition. Sports Medicine, 48(1), 7-16. PubMed ID: 28853006 doi:10.1007/ s40279-017-0776-1

Pickering, C., \& Kiely, J. (2019). What should we do about habitual caffeine use in athletes? Sports Medicine, 49(6), 833-842. PubMed ID: 30173351 doi:10.1007/s40279-018-0980-7

Poslusna, K., Ruprich, J., de Vries, J.H., Jakubikova, M., \& van’t Veer, P. (2009). Misreporting of energy and micronutrient intake estimated by food records and 24 hour recalls, control and adjustment methods in practice. British Journal of Nutrition, 101(Suppl. 2), S73-S85. doi:10.1017/S0007114509990602

Sabol, F., Grgic, J., \& Mikulic, P. (2019). The effects of 3 different doses of caffeine on jumping and throwing performance: A randomized, double-blind, crossover study. International Journal of Sports Physiology and Performance, 14(9), 1170-1177. doi:10.1123/ijspp.2018-0884

Scott, N.R., Chakraborty, J., \& Marks, V. (1984). Determination of caffeine, theophylline, and theobromine in serum and saliva using high performance liquid chromatography. Annals of Clinical Biochemistry, 21(2), 120-124. PubMed ID: 6712142 doi:10.1177/ 000456328402100208

Sökmen, B., Armstrong, L.E., Kraemer, W.J., Casa, D.J., Dias, J.C., Judelson, D.A., \& Maresh, C.M. (2008). Caffeine use in sports: 
Considerations for the athlete. The Journal of Strength and Conditioning Research, 22(3), 978-986. PubMed ID: 18438212 doi:10. 1519/JSC.0b013e3181660cec

Spriet, L.L. (2014). Exercise and sport performance with low doses of caffeine. Sports Medicine, 44(Suppl. 2), 175-184. doi:10.1007/ s40279-014-0257-8

Suzuki, Y., Uematsu, T., Mizuno, A., Fujii, K., \& Nakashima, M. (1989). Determination of caffeine in saliva by high-performance liquid chromatography: New sampling method for saliva using filter paper. Therapeutic Drug Monitoring, 11(1), 88-92. PubMed ID: 2911858 doi:10.1097/00007691-198901000-00018

Svenningsson, P., Nomikos, G.G., \& Fredholm, B.B. (1999). The stimulatory action and the development of tolerance to caffeine is associated with alterations in gene expression in specific brain regions. The Journal of Neuroscience, 19(10), 4011-4022. PubMed ID: 10234030 doi:10.1523/JNEUROSCI.19-10-04011. 1999

Tarnopolsky, M., \& Cupido, C. (2000). Caffeine potentiates low frequency skeletal muscle force in habitual and nonhabitual caffeine consumers. Journal of Applied Physiology, 89(5), 1719-1724. doi:10.1152/jappl. 2000.89.5.1719

Wiles, J.D., Bird, S.R., Hopkins, J., \& Riley, M. (1992). Effect of caffeinated coffee on running speed, respiratory factors, blood lactate and perceived exertion during 1500-m treadmill running. British Journal of Sports Medicine, 26(2), 116-120. PubMed ID: 1623356 doi:10.1136/bjsm.26.2.116 